\title{
Cycles and chaos in the one-sector growth model with elastic labor supply
}

\author{
Gerhard Sorger $^{1}(\mathbb{D}$
}

Received: 5 January 2016 / Accepted: 23 September 2016 / Published online: 1 October 2016 (C) The Author(s) 2016. This article is published with open access at Springerlink.com

\begin{abstract}
It is shown that the discrete-time version of the neoclassical one-sector optimal growth model with elastic labor supply and standard monotonicity and convexity assumptions on technology and preferences can have periodic solutions of any period as well as chaotic solutions. The optimality of these non-monotonic solutions is traced back to strong income effects. When technology and preferences are parameterized as it is commonly done in quantitative macroeconomic studies, these phenomena cannot occur.
\end{abstract}

Keywords Optimal growth · Endogenous labor supply · Periodic solutions · Chaotic dynamics

\section{JEL Classification C61 · O41}

\section{Introduction}

The neoclassical one-sector growth model with infinitely lived households has been widely used to study the causes and consequences of long-run growth and business cycles. Whereas the deterministic version of this model with inelastic labor supply has the property that all solutions converge monotonically to a unique interior steady state, this is not necessarily the case when labor supply is elastic and time is modeled as a discrete variable. De Hek (1998) provides both an example in which there exist multiple interior steady states and an example in which the optimal solution displays periodic oscillations. He concludes his paper by posing the question of "whether this model

$\bowtie$ Gerhard Sorger

gerhard.sorger@univie.ac.at

1 Department of Economics, University of Vienna, Oskar-Morgenstern-Platz 1, 1090 Vienna, Austria 
with leisure-dependent utility is able to generate more complex dynamics, in particular, chaos" (De Hek 1998, p. 270). Kamihigashi (2015) addresses the multiplicity of steady states in a more systematic way and proves that the model can have any finite number of steady states or even a continuum of steady states.

The purpose of the present paper is to conduct a similar systematic analysis of period-2 cycles and to provide an affirmative answer to the question by De Hek (1998) regarding more complicated dynamics. To this end, we first extend the results from De Hek (1998) by proving that, for any time-preference factor between 0 and 1, there exist a production function and an instantaneous utility function-both satisfying standard monotonicity and convexity assumptions_-such that the resulting model admits a locally asymptotically stable optimal solution which is periodic with period 2 . Furthermore, we construct a robust example of an economy for which there exists an optimal solution that has period 3. The existence of a solution with period 3 implies the existence of periodic solutions of all periods (see Sarkovskii 1964) as well as the occurrence of topological chaos (see Li and Yorke 1975).

The one-sector growth model with elastic labor supply forms the backbone of many modern dynamic stochastic general equilibrium models including real-business-cycle models and new Keynesian models. The standard approach to analyzing these models involves as its first step the specification of production functions and utility functions in parametric form. There are only a few classes of parametric families of functions that are used in quantitative macroeconomic research. In these specifications, it is typically the case that the utility function is additively separable with respect to consumption and leisure or that both the production function and the utility function are smooth with nonnegative cross-partial derivatives. We show that neither cycles nor chaos can be optimal under these assumptions. Moreover, the emergence of period-3 cycles requires unrealistically strong time preference as shown by Sorger (1994), Mitra (1996), and Nishimura and Yano (1996). Finally, we also point out that according to Hartl (1987) non-monotonic dynamics such as cycles and chaos are ruled out in the continuous-time version of the model.

The parametric families of production and utility functions mentioned in the previous paragraph form only a small subset of the set of those functions which satisfy the standard monotonicity and convexity assumptions on technology and preferences. Nevertheless, intuitive reasoning in the economics profession is highly influenced by models which use these parametric specifications. It is therefore of interest to find out which mechanisms are responsible for the periodic or even chaotic optimal solutions and which are therefore ruled out by the typical parametric specifications. De Hek (1998) links the occurrence of multiple steady states and cycles to the substitutability or complementarity, respectively, of consumption and leisure. Kamihigashi (2015), on the other hand, argues that normality of leisure is what rules out multiple steady states. The results and examples of the present paper support an intuitive explanation by income effects as in Kamihigashi (2015) rather than by substitution effects as in De Hek (1998). In particular, we believe that a strong and positive income effect on consumption combined with inferiority of leisure is the most plausible explanation of the non-monotonicity of optimal solutions. 
The model studied in the present paper coincides with that analyzed by Kamihigashi (2015) but differs slightly from that used by De Hek (1998). ${ }^{1}$ More specifically, we assume that output in period $t$ is produced from investment made in period $t-1$ and labor supplied in period $t$, whereas De Hek (1998) assumes that both factor inputs for period- $t$ production are determined in period $t-1$. We have chosen the present setting because we consider it to be more popular in macroeconomics than the one employed in De Hek (1998). It has to be emphasized, however, that for every result or example presented in this paper one can derive a corresponding result or example, respectively, using the timing assumption from De Hek (1998). In particular, deterministic chaos can also occur in the setting studied in De Hek (1998).

The rest of the paper is organized as follows. Section 2 describes the model and Sect. 3 presents and discusses the results and their implications. All proofs are given in "Appendix."

\section{The model}

Time evolves in discrete periods $t \in \mathbb{N}_{0}=\{0,1,2, \ldots\}$. Let us denote by $k_{t}$ and $\ell_{t}$ the period- $t$ factor inputs of capital and labor, respectively, and by $f\left(k_{t}, \ell_{t}\right)$ the amount of output that is available in period $t$. This amount consists of output produced in period $t$ plus non-depreciated capital from the previous period. The production function $f$ satisfies the following assumption.

Assumption 1 (i) The function $f: \mathbb{R}_{+} \times[0,1] \mapsto \mathbb{R}_{+}$is continuous, concave, homogeneous of degree 1 , and continuously differentiable on $\mathbb{R}_{++} \times(0,1]$.

(ii) There exists $\bar{k}>0$ such that $f(\bar{k}, 1)=\bar{k}$.

(iii) For every $k>0$, it holds that $f(k, \ell)$ is strictly increasing and strictly concave with respect to $\ell \in[0,1]$.

(iv) For every $\ell \in(0,1]$, it holds that $f(k, \ell)$ is strictly increasing and strictly concave with respect to $k \in \mathbb{R}_{+}$.

Output can be used for consumption and for investment, and we denote by $c_{t}$ the amount that is consumed in period $t$. A sequence $\left(k_{t}, \ell_{t}, c_{t}\right)_{t=0}^{+\infty}$ is called a feasible allocation if the conditions $k_{t} \geq 0, \ell_{t} \in[0,1], c_{t} \geq 0$, and

$$
c_{t}+k_{t+1}=f\left(k_{t}, \ell_{t}\right)
$$

hold for all $t \in \mathbb{N}_{0}$.

Throughout the paper, we assume without further mentioning that the initial endowment of the economy with capital, $k_{0}$, is such that $k_{0} \in[0, \bar{k}]$. Together with Assumption 1, this implies that every feasible allocation satisfies $k_{t} \in[0, \bar{k}]$ and $c_{t} \in[0, \bar{k}]$ for all $t \in \mathbb{N}_{0}$. When we specify the preferences over allocations, we may therefore restrict the domain of the instantaneous utility function accordingly.

The economy is endowed with a single unit of time per period such that $1-\ell_{t}$ denotes the time that is available for leisure. The preferences of the social planner are described by the welfare functional

\footnotetext{
${ }^{1}$ See also footnote 2 in Kamihigashi (2015).
} 


$$
\sum_{t=0}^{+\infty} \beta^{t} u\left(c_{t}, 1-\ell_{t}\right)
$$

where $u$ is an instantaneous utility function depending on consumption and leisure and $\beta$ is a time-preference factor.

Assumption 2 The function $u:[0, \bar{k}] \times[0,1] \mapsto \mathbb{R}$ is continuous, strictly increasing, and strictly concave.

Assumption 3 It holds that $\beta \in(0,1)$.

An economy is a triple $(f, u, \beta)$. Suppose that an economy $(f, u, \beta)$ and an initial capital endowment $\kappa \in[0, \bar{k}]$ are given. A feasible allocation $\left(k_{t}, \ell_{t}, c_{t}\right)_{t=0}^{+\infty}$ is said to be interior if $\left(k_{t}, \ell_{t}, c_{t}\right) \in(0, \bar{k}) \times(0,1) \times(0, \bar{k})$ holds for all $t \in \mathbb{N}_{0}$, and it is said to be optimal from $\kappa$ if it maximizes the welfare functional (2) over all feasible allocations with the given initial capital endowment $k_{0}=\kappa$. A feasible allocation $\left(k_{t}, \ell_{t}, c_{t}\right)_{t=0}^{+\infty}$ is called an optimal allocation for the economy $(f, u, \beta)$ if there exists an initial endowment $\kappa \in[0, \bar{k}]$ such that $\left(k_{t}, \ell_{t}, c_{t}\right)_{t=0}^{+\infty}$ is an optimal allocation from $\kappa$.

\section{The results and their implications}

In this section, we investigate under which conditions an economy $(f, u, \beta)$ admits optimal allocations which are periodic of period 2 or 3 . We will also explain why the consideration of these two period lengths is important.

We start with the case of period 2. A feasible allocation $\left(k_{t}, \ell_{t}, c_{t}\right)_{t=0}^{+\infty}$ is said to be periodic of period 2 if there exist real numbers $k_{a}, k_{b}, \ell_{a}, \ell_{b}, c_{a}$, and $c_{b}$ such that $\left(k_{i}, \ell_{i}, c_{i}\right) \in[0, \bar{k}] \times[0,1] \times[0, \bar{k}]$ for $i \in\{a, b\}, k_{a} \neq k_{b}$, and

$$
\left(k_{t}, \ell_{t}, c_{t}\right)= \begin{cases}\left(k_{a}, \ell_{a}, c_{a}\right) & \text { if } t \equiv 0 \bmod 2, \\ \left(k_{b}, \ell_{b}, c_{b}\right) & \text { if } t \equiv 1 \bmod 2\end{cases}
$$

hold. It will be convenient to introduce the following notation: ${ }^{2}$

$$
\begin{gathered}
F_{a}=f\left(k_{a}, \ell_{a}\right)+k_{a}-k_{b}-f_{1}\left(k_{b}, \ell_{b}\right) k_{b}-f_{2}\left(k_{b}, \ell_{b}\right) \ell_{a}, \\
F_{b}=f\left(k_{b}, \ell_{b}\right)+k_{b}-k_{a}-f_{1}\left(k_{a}, \ell_{a}\right) k_{a}-f_{2}\left(k_{a}, \ell_{a}\right) \ell_{b} .
\end{gathered}
$$

Theorem 1 Let $f$ and $\beta$ be given such that Assumptions 1 and 3 hold. Suppose furthermore that there exist real numbers $k_{a}, k_{b}, \ell_{a}$, and $\ell_{b}$ with $k_{a} \neq k_{b}$ such that

\footnotetext{
2 Throughout the paper, we denote partial derivatives by subscripts. For example, $u_{1}\left(c_{a}, 1-\ell_{a}\right)$ is the partial derivative of the instantaneous utility function $u$ with respect to its first argument evaluated at the point $\left(c_{a}, 1-\ell_{a}\right)$. Analogously, $f_{2}\left(k_{a}, \ell_{a}\right)$ is the partial derivative of the production function $f$ with respect to its second argument evaluated at the point $\left(k_{a}, \ell_{a}\right)$. The notation for higher-order partial derivatives is analogous: For example, $f_{12}\left(k_{a}, \ell_{a}\right)$ denotes the cross-partial derivative of $f$ at point $\left(k_{a}, \ell_{a}\right)$.
} 
$\left(k_{i}, \ell_{i}\right) \in(0, \bar{k}) \times(0,1)$ holds for $i \in\{a, b\}$. The following two statements are equivalent:

(a) There exist an instantaneous utility function $u$ satisfying Assumption 2 and a pair of real numbers $\left(c_{a}, c_{b}\right) \in(0, \bar{k})^{2}$ such that the periodic sequence defined by

(3) is an optimal allocation for the economy $(f, u, \beta)$.

(b) It holds that

$$
\begin{aligned}
& \beta^{2} f_{1}\left(k_{a}, \ell_{a}\right) f_{1}\left(k_{b}, \ell_{b}\right)=1, \\
& f\left(k_{a}, \ell_{a}\right)-k_{b}>0, \\
& f\left(k_{b}, \ell_{b}\right)-k_{a}>0, \\
& F_{b}+\beta f_{1}\left(k_{a}, \ell_{a}\right) F_{a}>0 .
\end{aligned}
$$

We will now show by means of an example that the conditions stated in part (b) of the theorem can be satisfied for any feasible value $\beta \in(0,1)$ even if one restricts the technology to be of Cobb-Douglas form.

Example 1 Let $\alpha \in(0,1)$ and $\beta \in(0,1)$ be arbitrary constants and define

$$
A=1 /(\alpha \beta)>1 \text {. }
$$

We consider a Cobb-Douglas production function of the form $f(k, \ell)=A k^{\alpha} \ell^{1-\alpha}$. Moreover, let $\gamma>1$ be given and assume that $k_{a}=\gamma \ell_{a}$ and $k_{b}=\gamma^{-1} \ell_{b}$. These definitions imply that $f_{1}\left(k_{a}, \ell_{a}\right)=\alpha A \gamma^{\alpha-1}, f_{1}\left(k_{b}, \ell_{b}\right)=\alpha A \gamma^{1-\alpha}$,

$$
F_{a}=\left[A \gamma^{\alpha}+\gamma-(1-\alpha) A \gamma^{-\alpha}\right] \ell_{a}-\left(\gamma^{-1}+\alpha A \gamma^{-\alpha}\right) \ell_{b},
$$

and

$$
F_{b}=\left[A \gamma^{-\alpha}+\gamma^{-1}-(1-\alpha) A \gamma^{\alpha}\right] \ell_{b}-\left(\gamma+\alpha A \gamma^{\alpha}\right) \ell_{a}
$$

Because of $A=1 /(\alpha \beta)$, it follows immediately that condition (4) is satisfied. It remains to be shown that one can find $\gamma>1$ such that (5)-(7) hold. Using the above expressions, we can rewrite these three inequalities as

$$
\begin{aligned}
& A \gamma^{\alpha} \ell_{a}>\gamma^{-1} \ell_{b}, \\
& A \gamma^{-\alpha} \ell_{b}>\gamma \ell_{a}, \\
& T_{a}(\gamma) \ell_{b}<T_{b}(\gamma) \ell_{a},
\end{aligned}
$$

where

$$
T_{a}(\gamma)=(1-\alpha) A \gamma^{\alpha}+\gamma^{\alpha-2}-(1-\alpha A) \gamma^{-1}-A \gamma^{-\alpha}
$$

and

$$
T_{b}(\gamma)=(1-\alpha A) \gamma^{\alpha}+A \gamma^{2 \alpha-1}-(1-\alpha) A \gamma^{-1}-\gamma
$$


Because of $A>1$, it follows that the two inequalities in (8)-(9) determine a nonempty conical area in $\left(\ell_{a}, \ell_{b}\right)$-space, the lower boundary of which is formed by the ray $\ell_{b}=A^{-1} \gamma^{1+\alpha} \ell_{a}$ and the upper boundary by the ray $\ell_{b}=A \gamma^{1+\alpha} \ell_{a}$. Using $A=1 /(\alpha \beta), \alpha \in(0,1)$, and $\beta \in(0,1)$, it is straightforward to verify that $T_{i}^{\prime}(1)>$ $0=T_{i}(1)$ holds for $i \in\{a, b\}$. Obviously, this implies that both $T_{a}(\gamma)$ and $T_{b}(\gamma)$ are strictly positive, provided that $\gamma$ is strictly greater than but sufficiently close to 1. Consequently, there exists a pair $\left(\ell_{a}, \ell_{b}\right) \in(0,1)^{2}$ such that the three inequalities (8)-(10) are simultaneously satisfied, provided that $T_{b}(\gamma) / T_{a}(\gamma)>A^{-1} \gamma^{1+\alpha}$. The latter condition can be expressed as

$$
\begin{aligned}
Z(\gamma)= & \left(1-\alpha-\alpha A+A^{-1}\right) \gamma^{\alpha}+\left(A-A^{-1}\right) \gamma^{2 \alpha-1}-(1-\alpha) A \gamma^{-1} \\
& -(1-\alpha) \gamma^{1+2 \alpha}>0
\end{aligned}
$$

We have $Z(1)=0$ and

$$
Z^{\prime}(1)=-\alpha^{2} A+\alpha^{2}-\alpha A^{-1}+\alpha A+A^{-1}-1=(1-\alpha)(1-\beta)(1-\alpha \beta) / \beta>0,
$$

where we have once more used $A=1 /(\alpha \beta), \alpha \in(0,1)$, and $\beta \in(0,1)$. Obviously, this implies that $Z(\gamma)>0$ holds for $\gamma$ greater than but sufficiently close to 1 . Hence, we have demonstrated that, for every time-preference factor $\beta \in(0,1)$, there exist a Cobb-Douglas production function $f$ and numbers $k_{a}, k_{b}, \ell_{a}, \ell_{b}$ with $k_{a} \neq k_{b}$ such that condition (b) of Theorem 1 holds.

Together with Theorem 1, the above example gives rise to the following corollary.

Corollary 1 For every $\beta \in(0,1)$, there exist a Cobb-Douglas production function $f$ and an instantaneous utility function $u$ satisfying Assumption 2 such that the economy $(f, u, \beta)$ admits an optimal allocation which is periodic of period 2.

In "Appendix," we show that the periodic optimal allocations constructed in Example 1 are locally asymptotically stable, provided that the utility function $u$ is chosen appropriately. Hence, our results are more general than those in De Hek (1998) as they guarantee the existence of locally asymptotically stable optimal allocations of period 2 for all time-preference factors $\beta \in(0,1)$.

Even if Corollary 1 demonstrates that periodic optimal allocations can exist under a typical parametric specification of the production function (namely Cobb-Douglas), it does not say anything about the properties of the utility function that give rise to such an outcome. Our next result identifies two conditions that rule out the optimality of periodic allocations.

Theorem 2 Consider an economy $(f, u, \beta)$ satisfying Assumptions 1-3 and suppose that there exist real numbers $k_{a}, k_{b}, \ell_{a}, \ell_{b}, c_{a}$, and $c_{b}$ satisfying $k_{a} \neq k_{b}$ and $\left(k_{i}, \ell_{i}, c_{i}\right) \in(0, \bar{k}) \times(0,1) \times(0, \bar{k})$ for $i \in\{a, b\}$ such that the periodic sequence defined by (3) is an optimal allocation for the economy $(f, u, \beta)$. Then neither of the following two statements is true:

(a) There exist functions $v:[0, \bar{k}] \mapsto \mathbb{R}$ and $q:[0,1] \mapsto \mathbb{R}$ such that $u(c, 1-\ell)=$ $v(c)+q(1-\ell)$ holds for all $(c, \ell) \in[0, \bar{k}] \times[0,1]$ and such that $v$ and $q$ are locally differentiable at $c_{i}$ and $1-\ell_{i}$, respectively, for both $i \in\{a, b\}$. 
(b) The functions $f$ and $u$ are twice continuously differentiable with $f_{12}(k, \ell) \geq 0$ and $u_{12}(c, 1-\ell) \geq 0$ for all $(k, \ell, c) \in(0, \bar{k}] \times(0,1) \times(0, \bar{k}]$.

Theorem 2(a) is related to corollary 2 in Kamihigashi (2015), which shows that in the case of an additively separable utility function the model admits at most one interior steady state. Theorem 2(b) is related both to proposition 1 in Kamihigashi (2015) and to proposition 2.4 in De Hek (1998). To explain these relationships, first note that $u_{12}(c, 1-\ell) \geq 0$ for all $(c, \ell) \in(0, \bar{k}) \times(0,1)$ is a sufficient condition for the normality of both consumption and leisure. This observation provides the link to proposition 1 in Kamihigashi (2015), which is directly derived from the assumption of normality of leisure. The connection to proposition 2.4 in De Hek (1998) becomes clear by noting that De Hek (1998) assumes that output in period $t$ is produced from labor supplied in period $t-1$, whereas we assume that output in period $t$ requires labor input in the same period $t$. This difference in timing has the effect that Theorem 2(b) above requires a nonnegative cross-partial derivative $u_{12}(c, 1-\ell)$, whereas De Hek (1998) can rule out periodic optimal allocations in the case where $u_{12}(c, 1-\ell) \leq 0$ holds.

Since most of the parametric families of production and utility functions used in quantitative macroeconomic models satisfy at least one of the two properties mentioned in Theorem 2, we cannot expect to find familiar closed-form utility functions that allow periodic optimal allocations. In the proof of Theorem 1, we construct the utility function as the pointwise minimum of two quadratic polynomials in consumption and leisure. Properties of such a utility function (in addition to those that are imposed by Assumption 2) are hard to characterize. The advantage of the construction in the proof of Theorem 1 is that it can easily be modified to find economies with optimal allocations of any period, see Theorem 3 and the ensuing discussion. In the present case of an optimal allocation of period 2, however, there does indeed exist a simpler construction which results in a quadratic utility function. We illustrate this construction in the following example, which will also be helpful to understand the mechanisms that are responsible for the optimality of periodic allocations. ${ }^{3}$

Example 2 As in Example 1, we assume $f(k, \ell)=A k^{\alpha} \ell^{1-\alpha}, A=1 /(\alpha \beta), k_{a}=\gamma \ell_{a}$, and $k_{b}=\gamma^{-1} \ell_{b}$, where $\gamma>1$. Let us fix the parameter values $\alpha=\beta=1 / 2$, from which it follows that $A=4$ and $\bar{k}=16$. Furthermore, we set $\ell_{a}=1 / 2$ and $\ell_{b}=1 / 3$. We know from Example 1 that, whenever $\gamma$ is sufficiently close to 1 , there exist a utility function $u$ satisfying Assumption 2 and consumption values $c_{a}$ and $c_{b}$ such that the economy $(f, u, \beta)$ admits the optimal allocation defined by $\left(k_{a}, \ell_{a}, c_{a}\right)$ and $\left(k_{b}, \ell_{b}, c_{b}\right)$. We shall now demonstrate that the quadratic utility function

$$
u(c, 1-\ell)=p_{1} c+p_{2}(1-\ell)+\frac{p_{11} c^{2}}{2}+p_{12} c(1-\ell)+\frac{p_{22}(1-\ell)^{2}}{2}
$$

does the trick, provided that the coefficients $p_{1}, p_{2}, p_{11}, p_{12}$, and $p_{22}$ are chosen appropriately.

\footnotetext{
3 I am indebted to Marwan Lisser for making me aware of this simpler construction in the case of period 2.
} 
Using the above specifications and defining $z=\gamma^{1 / 2}$, we have

$$
\begin{aligned}
& k_{a}=\frac{z^{2}}{2}, \quad \ell_{a}=\frac{1}{2}, \quad c_{a}=2 z-\frac{1}{3 z^{2}}, \quad k_{b}=\frac{1}{3 z^{2}}, \quad \ell_{b}=\frac{1}{3}, \quad c_{b}=\frac{4}{3 z}-\frac{z^{2}}{2}, \\
& f\left(k_{a}, \ell_{a}\right)=2 z, \quad f\left(k_{b}, \ell_{b}\right)=\frac{4}{3 z},
\end{aligned}
$$

and

$$
f_{1}\left(k_{a}, \ell_{a}\right)=f_{2}\left(k_{b}, \ell_{b}\right)=\frac{2}{z}, f_{2}\left(k_{a}, \ell_{a}\right)=f_{1}\left(k_{b}, \ell_{b}\right)=2 z
$$

Substituting (11)-(13) into conditions (42)-(45) in "Appendix," we obtain four equations for the five undetermined coefficients $p_{1}, p_{2}, p_{11}, p_{12}$, and $p_{22}$. One set of values satisfying these equations is given by

$$
\begin{aligned}
p_{1} & =\frac{21}{20}, \\
p_{2} & =\frac{40-162 z-8 z^{2}-240 z^{3}+412 z^{4}+3 z^{5}}{20 z^{3}}, \\
p_{11} & =\frac{3 z(60-59 z)}{10\left(8+24 z-48 z^{3}-9 z^{4}\right)}, \\
p_{12} & =\frac{3\left(40-42 z-8 z^{2}-240 z^{3}+252 z^{4}+3 z^{5}\right)}{10 z\left(8+24 z-48 z^{3}-9 z^{4}\right)}, \\
p_{22} & =-\frac{3 P(z)}{10 z^{3}\left(8+24 z-48 z^{3}-9 z^{4}\right)},
\end{aligned}
$$

where

$$
\begin{aligned}
P(z)= & 80-84 z-1312 z^{2}-1024 z^{3}+1208 z^{4}+3564 z^{5}+2928 z^{6}-4224 z^{7} \\
& -1152 z^{8}-9 z^{9}
\end{aligned}
$$

It is straightforward to verify that the function $u$ in (11) satisfies Assumption 2 whenever $z>1$ is chosen sufficiently close to 1 . The optimality of the periodic allocation defined by $\left(k_{a}, \ell_{a}, c_{a}\right)$ and $\left(k_{b}, \ell_{b}, c_{b}\right)$ for such a value of $z$ follows now directly from the fact that (42)-(45) hold and from Lemma 1 in "Appendix."

Let us try to develop some intuition for the emergence of non-monotonic dynamics in the one-sector growth model with elastic labor supply. De Hek (1998) attributes this phenomenon to consumption and labor being complements. What he means by that is the property that $u_{12}(c, 1-\ell)>0$ holds. ${ }^{4}$ As we have pointed out before, the different timing assumption in our model would then mean that periodic optimal allocations require consumption and leisure to be substitutes in the sense of $u_{12}(c, 1-\ell)<0$,

\footnotetext{
${ }^{4}$ This is not the usual definition of complementarity, though. According to Shephard's lemma, two goods are (net) complements if the cross-partial derivative of the expenditure function with respect to the prices is negative rather than if the cross-partial derivative of the utility function with respect to the quantities is positive.
} 
which is indeed consistent with Theorem 2 . This property alone, however, does not seem to be the whole explanation. To explain our reasoning, consider Example 2 in the limit as $z=\gamma^{1 / 2}$ approaches 1 . The utility function and the allocation in this limit are as follows: ${ }^{5}$

$$
\begin{aligned}
& u(c, 1-\ell)=\frac{21 c}{20}+\frac{9(1-\ell)}{4}-\frac{3 c^{2}}{500}-\frac{3 c(1-\ell)}{50}-\frac{3(1-\ell)^{2}}{20}, \\
& k_{a}=\frac{1}{2}, \quad \ell_{a}=\frac{1}{2}, \quad c_{a}=\frac{5}{3}, \quad k_{b}=\frac{1}{3}, \quad \ell_{b}=\frac{1}{3}, \quad c_{b}=\frac{5}{6}, \\
& f\left(k_{a}, \ell_{a}\right)=2, \quad f\left(k_{b}, \ell_{b}\right)=\frac{4}{3}, \\
& f_{1}\left(k_{a}, \ell_{a}\right)=f_{2}\left(k_{a}, \ell_{a}\right)=f_{1}\left(k_{b}, \ell_{b}\right)=f_{2}\left(k_{b}, \ell_{b}\right)=2 .
\end{aligned}
$$

First note from (17) that the marginal products $f_{1}\left(k_{t}, \ell_{t}\right)$ and $f_{2}\left(k_{t}, \ell_{t}\right)$ are constant along the periodic allocation. In a competitive equilibrium interpretation of the model, these marginal products correspond to the factor prices. The fact that the factor prices are constant along the allocation suggests that neither intertemporal nor intratemporal substitution effects are responsible for the non-monotonic dynamics. As we will argue now, it is much more plausible that income effects play a major role. Given the quadratic utility function in (14), it is easy to compute the corresponding demand functions which maximize $u(c, 1-\ell)$ subject to the budget constraint $c+\omega(1-\ell) \leq y$, where $y$ denotes income and $\omega$ is the price of leisure relative to consumption. In the current model, we know that the relative price $\omega$ must be equal to the marginal product of labor, that is, $\omega=f_{2}(k, \ell)$. We see from (17) that this relative price is constant and equal to 2 along the periodic allocation under consideration. Because the utility function is quadratic, the demand functions are linear in income $y$, and the coefficients of $y$ in the demand functions for consumption or leisure measure the effects of income changes on the demand for the respective good. In the demand function for consumption, this coefficient is equal to $5 / 3$, whereas in the demand function for leisure it is $-1 / 3$. We therefore see that leisure is an inferior good. What is more important, however, is that consumption reacts very strongly to income changes. Now consider the periodic optimal allocation in (15). If the economy is in state $a$ with the high capital stock $k_{a}=1 / 2$, income is high as well, namely $f\left(k_{a}, \ell_{a}\right)=2$. Due to the strong income effect on consumption, consumption in state $a$ is $c_{a}=5 / 3$, which corresponds to more than $83 \%$ of income. This, in turn, means that households save very little and that next period's capital stock and income are low, namely $k_{b}=1 / 3$ and $f\left(k_{b}, \ell_{b}\right)=4 / 3$. The strong (positive) income effect on consumption implies that consumption in state $b$ is $c_{b}=5 / 6$, a mere $62.5 \%$ of income. The corresponding high investment brings the capital stock back up to $k_{a}=1 / 2$, and the same process repeats itself. Inferiority of leisure amplifies the fluctuations, because it leads to high (low) labor input in periods with high (low) capital. We believe that these mechanisms, which are clearly visible in Example 2, are also the main driving forces for the non-monotonicity of allocations in the general model.

\footnotetext{
5 For $z$ slightly larger than 1, the values will be close to those stated in (14)-(17).
} 
Let us now turn to the case of period 3. A feasible allocation $\left(k_{t}, \ell_{t}, c_{t}\right)_{t=0}^{+\infty}$ is periodic of period 3 if there exist real numbers $k_{a}, k_{b}, k_{c}, \ell_{a}, \ell_{b}, \ell_{c}, c_{a}, c_{b}$, and $c_{c}$ such that $k_{a}, k_{b}$, and $k_{c}$ are mutually different from each other and such that

$$
\left(k_{t}, \ell_{t}, c_{t}\right)= \begin{cases}\left(k_{a}, \ell_{a}, c_{a}\right) & \text { if } t \equiv 0 \bmod 3, \\ \left(k_{b}, \ell_{b}, c_{b}\right) & \text { if } t \equiv 1 \bmod 3 \\ \left(k_{c}, \ell_{c}, c_{c}\right) & \text { if } t \equiv 2 \bmod 3\end{cases}
$$

holds. Given such an allocation, we define

$$
\begin{aligned}
& F_{a c}=f\left(k_{a}, \ell_{a}\right)+k_{c}-k_{b}-f_{1}\left(k_{b}, \ell_{b}\right) k_{b}-f_{2}\left(k_{b}, \ell_{b}\right) \ell_{a}, \\
& F_{b a}=f\left(k_{b}, \ell_{b}\right)+k_{a}-k_{c}-f_{1}\left(k_{c}, \ell_{c}\right) k_{c}-f_{2}\left(k_{c}, \ell_{c}\right) \ell_{b}, \\
& F_{c b}=f\left(k_{c}, \ell_{c}\right)+k_{b}-k_{a}-f_{1}\left(k_{a}, \ell_{a}\right) k_{a}-f_{2}\left(k_{a}, \ell_{a}\right) \ell_{c}, \\
& F_{a a}=f\left(k_{a}, \ell_{a}\right)+k_{a}-k_{b}-f_{1}\left(k_{c}, \ell_{c}\right) k_{c}-f_{2}\left(k_{c}, \ell_{c}\right) \ell_{a}, \\
& F_{b b}=f\left(k_{b}, \ell_{b}\right)+k_{b}-k_{c}-f_{1}\left(k_{a}, \ell_{a}\right) k_{a}-f_{2}\left(k_{a}, \ell_{a}\right) \ell_{b}, \\
& F_{c c}=f\left(k_{c}, \ell_{c}\right)+k_{c}-k_{a}-f_{1}\left(k_{b}, \ell_{b}\right) k_{b}-f_{2}\left(k_{b}, \ell_{b}\right) \ell_{c} .
\end{aligned}
$$

Theorem 3 Let $f$ and $\beta$ be given such that Assumptions 1 and 3 hold. Suppose furthermore that there exist real numbers $k_{a}, k_{b}, k_{c}, \ell_{a}, \ell_{b}$, and $\ell_{c}$ such that $k_{a} \neq k_{b}$, $k_{b} \neq k_{c}, k_{a} \neq k_{c}$, and $\left(k_{i}, \ell_{i}\right) \in(0, \bar{k}) \times(0,1)$ hold for $i \in\{a, b, c\}$. The following two statements are equivalent:

(a) There exist an instantaneous utility function $u$ satisfying Assumption 2 and a triple of real numbers $\left(c_{a}, c_{b}, c_{c}\right) \in(0, \bar{k})^{3}$ such that the periodic sequence defined by (18) is an optimal allocation for the economy $(f, u, \beta)$.

(b) It holds that

$$
\begin{aligned}
& \beta^{3} f_{1}\left(k_{a}, \ell_{a}\right) f_{1}\left(k_{b}, \ell_{b}\right) f_{1}\left(k_{c}, \ell_{c}\right)=1, \\
& f\left(k_{a}, \ell_{a}\right)-k_{b}>0, \\
& f\left(k_{b}, \ell_{b}\right)-k_{c}>0, \\
& f\left(k_{c}, \ell_{c}\right)-k_{a}>0, \\
& \beta^{2} f_{1}\left(k_{a}, \ell_{a}\right) f_{1}\left(k_{c}, \ell_{c}\right) F_{a c}+F_{b b}>0, \\
& \beta f_{1}\left(k_{c}, \ell_{c}\right) F_{c c}+F_{b a}>0, \\
& \beta f_{1}\left(k_{a}, \ell_{a}\right) F_{a a}+F_{c b}>0, \\
& \beta^{2} f_{1}\left(k_{a}, \ell_{a}\right) f_{1}\left(k_{c}, \ell_{c}\right) F_{c c}+\beta f_{1}\left(k_{a}, \ell_{a}\right) F_{a a}+F_{b b}>0, \\
& \beta^{2} f_{1}\left(k_{a}, \ell_{a}\right) f_{1}\left(k_{c}, \ell_{c}\right) F_{a c}+\beta f_{1}\left(k_{a}, \ell_{a}\right) F_{b a}+F_{c b}>0 .
\end{aligned}
$$

Having derived the necessary and sufficient conditions on $f$ and $\beta$ for the existence of an economy $(f, u, \beta)$ that admits an interior optimal allocation of period 3 , we now 
show by means of an example that these conditions can be satisfied even if we restrict the technology to be of the Cobb-Douglas variety. ${ }^{6}$

Example 3 We specify the production function by $f(k, \ell)=A k^{\alpha} \ell^{1-\alpha}$ with $\alpha=1 / 3$ and the time-preference factor by $\beta=7 / 20$. Furthermore, we choose the allocation variables $k_{a}=1507, k_{b}=2143, k_{c}=3200, \ell_{a}=2 / 5, \ell_{b}=57 / 100$, and $\ell_{c}=$ $21 / 25$. The productivity parameter $A$ is determined in such a way that (19) holds, which yields $A \approx 2079$. With these specifications, it is straightforward to verify that conditions (20)-(27) are satisfied. ${ }^{7}$ In particular, consumption is given by $c_{a} \approx 10800$, $c_{b} \approx 15231$, and $c_{c} \approx 25774$. Note that the periodic optimal allocation is apparently not close to a steady state because the coefficients of variation of capital, labor, and consumption along the allocation are between 30 and $40 \%$.

From Theorem 3 and Example 3, we obtain the following corollary.

Corollary 2 There exist a Cobb-Douglas production function $f$, an instantaneous utility function $u$ satisfying Assumption 2, and a time-preference factor $\beta$ satisfying Assumption 3 such that the economy $(f, u, \beta)$ admits an optimal allocation which is periodic of period 3.

To explain the implications of the above results, let an economy $(f, u, \beta)$ be given such that Assumptions 1-3 hold. It follows from standard results on dynamic program$\operatorname{ming}^{8}$ that, for every initial capital endowment $\kappa \in[0, \bar{k}]$, there exists a unique optimal allocation from $\kappa$. Moreover, there exists a continuous function $h:[0, \bar{k}] \mapsto[0, \bar{k}]$ such that the set of all capital sequences corresponding to optimal allocations for the economy $(f, u, \beta)$ coincides with the set of all trajectories of the difference equation

$$
k_{t+1}=h\left(k_{t}\right) \text { for all } t \in \mathbb{N}_{0}
$$

which start in initial states $k_{0} \in[0, \bar{k}]$. Equation (28) says that the capital stocks in every optimal allocation form a trajectory of a continuous dynamical system defined on the one-dimensional compact state space $[0, \bar{k}]$. When the economy $(f, u, \beta)$ admits an optimal allocation of period 3, it follows that the corresponding difference Eq. (28) has a periodic trajectory with period 3. Continuous dynamical systems which are defined on a one-dimensional state space, such as (28), are very well studied, and it is known that the existence of periodic solutions with period 3 has strong implications. ${ }^{9}$ First, according to Sarkovskii (1964) it follows that a difference equation that admits a periodic solution of period 3 admits periodic solutions of all periods $p \in \mathbb{N}$. Second, according to Li and Yorke (1975), the existence of a periodic solution of period 3 implies that the dynamical system (28) exhibits topological chaos. This means in particular that there exists an uncountable set $S$ of initial capital endowments such that the unique optimal allocation emanating from any $\kappa \in S$ is neither periodic nor

\footnotetext{
6 This is just one of several examples that we have found.

7 The verification has been executed in exact algebra with the software Mathematica ${ }^{\circledR}$.

8 See, e.g., Miao (2014), Sorger (2015), or Stokey and Lucas (1989).

9 See, e.g., Collet and Eckmann (1980), De Melo and Van Strien (1993), or Sorger (2015).
} 
asymptotically periodic and that, for any pair $\left\{\kappa, \kappa^{\prime}\right\} \subseteq S$, the two optimal allocations starting in $\kappa$ and $\kappa^{\prime}$, respectively, become arbitrarily close to each other without converging to each other. Hence, Corollary 2 proves that the neoclassical one-sector growth model with elastic labor supply can generate very complicated dynamics and that its optimal allocations can display sensitive dependence on initial conditions.

One consequence of Sarkovskii (1964) is that any economy $(f, u, \beta)$ that admits an optimal allocation of period 3 must also admit an optimal allocation of period 2. Hence, it follows from Theorem 2 that such an economy can have neither an additively separable utility function nor smooth production and utility functions with nonnegative cross-partial derivatives. Moreover, whereas we could establish the existence of optimal allocations of period 2 for any feasible time-preference factor $\beta \in(0,1)$, this is not possible in the case of optimal allocations of period 3. This follows from Mitra (1996) and Nishimura and Yano (1996), who have shown in a more general context that an optimal allocation with period 3 can only exist if $\beta<(3-\sqrt{5}) / 2 \approx 0.38$.

The mechanisms that generate chaotic dynamics in the present model are most likely the same ones that are responsible for the emergence of periodic allocations of period 2, namely strong income effects. These are consistent with the standard Assumptions 1-3, but they do not occur when production and utility functions are parameterized as it is commonly done in quantitative macroeconomic studies. In the constructive proof of Theorem 3, we define the utility function $u$ as the minimum of three quadratic polynomials, which does not lend itself to intuitive interpretations. The simpler construction used in Example 2 does not work in the present setting because a quadratic polynomial has only five parameters, which leaves too few degrees of freedom to satisfy the six equations stated in (69)-(74).

We have imposed smoothness of the production function $f$ in Assumption 1 but have not made an analogous assumption on the instantaneous utility function $u$. The reason is that the function $u$ employed in the constructive proofs of Theorems 1 and 3 , respectively, is the minimum of finitely many smooth polynomials. This function is not differentiable along the one-dimensional manifolds at which the graphs of the polynomials intersect. It is possible, however, to replace the function $u$ in these proofs by a smooth function satisfying Assumption 2 without violating any of the first-order conditions. This is the case because the first-order conditions involve the partial derivatives of the function $u$ only at the (two or three) points along the allocation, and because these points are separated from the manifolds along which the non-differentiability occurs. Thus, the two theorems would remain true if we were to strengthen Assumption 2 by imposing smoothness of the instantaneous utility function.

Finally let us point out that non-monotonic allocations such as periodic and chaotic ones cannot occur in a continuous-time version of the model such as the one underlying Benhabib and Farmer (1994) or Sorger (2000). This is the case because strict concavity of the utility function would imply the uniqueness of an optimal allocation from any given initial value $\kappa$ and because continuous-time dynamic optimization problems with a single state variable and unique optimal solutions cannot have non-monotonic optimal solutions (see, Hartl 1987). Thus, whereas the results about the possible multiplicity of steady states derived by Kamihigashi (2015) hold essentially unchanged if time varies continuously, our existence results for periodic optimal allocations have no counterpart in the continuous-time setting. 
Acknowledgements Open access funding provided by [University of Vienna]. The author thanks an associate editor and a referee for their encouraging and constructive comments that helped to considerably improve the paper.

\section{Compliance with ethical standards}

Conflicts of interest The author declares that he has no conflict of interest.

Open Access This article is distributed under the terms of the Creative Commons Attribution 4.0 International License (http://creativecommons.org/licenses/by/4.0/), which permits unrestricted use, distribution, and reproduction in any medium, provided you give appropriate credit to the original author(s) and the source, provide a link to the Creative Commons license, and indicate if changes were made.

\section{Appendix}

Lemma 1 Let $(f, u, \beta)$ be an economy satisfying Assumptions 1-3 and suppose that there exist real numbers $k_{a}, k_{b}, \ell_{a}, \ell_{b}, c_{a}$, and $c_{b}$ such that $k_{a} \neq k_{b}$ and $\left(k_{i}, \ell_{i}, c_{i}\right) \in$ $(0, \bar{k}) \times(0,1) \times(0, \bar{k})$ for $i \in\{a, b\}$ hold and such that the utility function $u$ is continuously differentiable locally around both of the two points $\left(c_{a}, 1-\ell_{a}\right)$ and $\left(c_{b}, 1-\ell_{b}\right)$.

(a) The sequence $\left(k_{t}, \ell_{t}, c_{t}\right)_{t=0}^{+\infty}$ defined by (3) is an optimal allocation for the economy $(f, u, \beta)$ if and only if the following conditions hold:

$$
\begin{aligned}
& c_{a}+k_{b}=f\left(k_{a}, \ell_{a}\right), \\
& c_{b}+k_{a}=f\left(k_{b}, \ell_{b}\right), \\
& \beta^{2} f_{1}\left(k_{a}, \ell_{a}\right) f_{1}\left(k_{b}, \ell_{b}\right)=1, \\
& u_{1}\left(c_{b}, 1-\ell_{b}\right)=\beta f_{1}\left(k_{a}, \ell_{a}\right) u_{1}\left(c_{a}, 1-\ell_{a}\right), \\
& u_{1}\left(c_{a}, 1-\ell_{a}\right) f_{2}\left(k_{a}, \ell_{a}\right)=u_{2}\left(c_{a}, 1-\ell_{a}\right), \\
& u_{1}\left(c_{b}, 1-\ell_{b}\right) f_{2}\left(k_{b}, \ell_{b}\right)=u_{2}\left(c_{b}, 1-\ell_{b}\right) .
\end{aligned}
$$

(b) If the sequence $\left(k_{t}, \ell_{t}, c_{t}\right)_{t=0}^{+\infty}$ defined by (3) is an optimal allocation for the economy $(f, u, \beta)$, then it holds that $\left(c_{a}, \ell_{a}\right) \neq\left(c_{b}, \ell_{b}\right)$.

Proof (a) Consider the allocation specified by (3). Because of $\left(k_{i}, \ell_{i}, c_{i}\right) \in(0, \bar{k}) \times$ $(0,1) \times(0, \bar{k})$, the allocation is feasible and interior if and only if (29)-(30) hold. It is known that, under Assumptions 1-3, an interior feasible allocation is an optimal allocation if and only if the first-order optimality conditions

$$
\begin{aligned}
& u_{1}\left(c_{t}, 1-\ell_{t}\right)=\beta f_{1}\left(k_{t+1}, \ell_{t+1}\right) u_{1}\left(c_{t+1}, 1-\ell_{t+1}\right) \quad \text { for all } t \in \mathbb{N}_{0}, \\
& u_{1}\left(c_{t}, 1-\ell_{t}\right) f_{2}\left(k_{t}, \ell_{t}\right)=u_{2}\left(c_{t}, 1-\ell_{t}\right) \text { for all } t \in \mathbb{N}_{0}
\end{aligned}
$$

as well as the transversality condition $\lim _{t \rightarrow+\infty} \beta^{t} u_{1}\left(c_{t}, 1-\ell_{t}\right) k_{t+1}=0$ are satisfied. These conditions require differentiability of the production function $f$ and the instantaneous utility function $u$ locally around the allocation, which has been assumed for $f$ in Assumption 1 and for $u$ directly in the lemma. The first-order condition (36) holds along the given allocation if and only if (33)-(34) are satisfied. The Euler Eq. (35) 
holds along the given allocation if and only if (32) and the corresponding equation

$$
u_{1}\left(c_{a}, 1-\ell_{a}\right)=\beta f_{1}\left(k_{b}, \ell_{b}\right) u_{1}\left(c_{b}, 1-\ell_{b}\right)
$$

are satisfied. Multiplying the left-hand sides and the right-hand sides of (32) and (37), we obtain (31). Conversely, if (31) holds, then one of the two Euler Eqs. (32) and (37) is redundant. In other words, Eqs. (32) and (37) together are equivalent to Eqs. (31)-(32). Finally, the transversality condition holds because of the boundedness of the allocation, the interiority of $\left(c_{i}, 1-\ell_{i}\right)$ for $i \in\{a, b\}$, and Assumption 3. This completes the proof of part (a).

(b) Suppose to the contrary that $\left(c_{a}, \ell_{a}\right)=\left(c_{b}, \ell_{b}\right)=(c, \ell)$ holds. Then it follows from (31)-(32) that $f_{1}\left(k_{a}, \ell\right)=f_{1}\left(k_{b}, \ell\right)=1 / \beta$, which contradicts $k_{a} \neq k_{b}$ due to the strict concavity of the mapping $k \mapsto f(k, \ell)$. The proof of the lemma is now complete.

Lemma 2 Let $X$ and $Y$ be non-empty and compact intervals on the real line and let $x_{a}, x_{b}, y_{a}$, and $y_{b}$ be real numbers such that $x_{i} \in$ int $X$ and $y_{i} \in$ int $Y$ hold for $i \in\{a, b\}$ and such that $\left(x_{a}, y_{a}\right) \neq\left(x_{b}, y_{b}\right)$. Furthermore, let $w_{1 a}, w_{1 b}, w_{2 a}$, and $w_{2 b}$ be positive real numbers. The following two statements are equivalent:

(a) There exists a function $w: X \times Y \mapsto \mathbb{R}$ which is continuous, strictly increasing, and strictly concave and which is continuously differentiable locally at the points $\left(x_{a}, y_{a}\right)$ and $\left(x_{b}, y_{b}\right)$ with partial derivatives $w_{1}\left(x_{i}, y_{i}\right)=w_{1 i}$ and $w_{2}\left(x_{i}, y_{i}\right)=w_{2 i}$ for $i \in\{a, b\}$.

(b) The inequality

$$
\left(w_{1 a}-w_{1 b}\right)\left(x_{b}-x_{a}\right)+\left(w_{2 a}-w_{2 b}\right)\left(y_{b}-y_{a}\right)>0
$$

holds.

Proof We first prove that (a) implies (b). By strict concavity of $w$ and $\left(x_{a}, y_{a}\right) \neq$ $\left(x_{b}, y_{b}\right)$, it follows that

$$
w\left(x_{a}, y_{a}\right)<w\left(x_{b}, y_{b}\right)+w_{1 b}\left(x_{a}-x_{b}\right)+w_{2 b}\left(y_{a}-y_{b}\right)
$$

and

$$
w\left(x_{b}, y_{b}\right)<w\left(x_{a}, y_{a}\right)+w_{1 a}\left(x_{b}-x_{a}\right)+w_{2 a}\left(y_{b}-y_{a}\right) .
$$

Combining these two inequalities, we obtain

$$
\begin{aligned}
& w_{1 b}\left(x_{b}-x_{a}\right)+w_{2 b}\left(y_{b}-y_{a}\right)<w\left(x_{b}, y_{b}\right)-w\left(x_{a}, y_{a}\right)<w_{1 a}\left(x_{b}-x_{a}\right) \\
& \quad+w_{2 a}\left(y_{b}-y_{a}\right) .
\end{aligned}
$$

Obviously, this implies (38).

The proof that (b) implies (a) is divided into three steps. 
Step 1: Inequality (38) is equivalent to $w_{1 b}\left(x_{b}-x_{a}\right)+w_{2 b}\left(y_{b}-y_{a}\right)<w_{1 a}\left(x_{b}-\right.$ $\left.x_{a}\right)+w_{2 a}\left(y_{b}-y_{a}\right)$. Hence, there exist real numbers $w_{a}$ and $w_{b}$ such that

$$
w_{1 b}\left(x_{b}-x_{a}\right)+w_{2 b}\left(y_{b}-y_{a}\right)<w_{b}-w_{a}<w_{1 a}\left(x_{b}-x_{a}\right)+w_{2 a}\left(y_{b}-y_{a}\right) \text {. }
$$

Step 2: We define for $i \in\{a, b\}$ and all $\left(\varepsilon_{1 i}, \varepsilon_{2 i}\right) \in \mathbb{R}_{+}^{2}$ the quadratic polynomial $g\left(\cdot, \cdot \mid i, \varepsilon_{1 i}, \varepsilon_{2 i}\right): X \times Y \mapsto \mathbb{R}$ by

$$
\begin{aligned}
& g\left(x, y \mid i, \varepsilon_{1 i}, \varepsilon_{2 i}\right)=w_{i}+w_{1 i}\left(x-x_{i}\right)+w_{2 i}\left(y-y_{i}\right) \\
& -\varepsilon_{1 i}\left(x-x_{i}\right)^{2}-\varepsilon_{2 i}\left(y-y_{i}\right)^{2} .
\end{aligned}
$$

Since the numbers $w_{1 i}$ and $w_{2 i}$ are strictly positive for $i \in\{a, b\}$, it follows that $g(x, y \mid i, 0,0)$ is strictly increasing with respect to $(x, y)$ for $i \in\{a, b\}$. Since $X \times Y$ is compact, this property is robust to small perturbations of $\varepsilon_{1 i}$ and $\varepsilon_{2 i}$. Hence, $g\left(x, y \mid i, \varepsilon_{1 i}, \varepsilon_{2 i}\right)$ is strictly increasing in $(x, y)$ for all sufficiently small positive numbers $\varepsilon_{1 i}$ and $\varepsilon_{2 i}$ and both $i \in\{a, b\}$. It is also clear that $g\left(x, y \mid i, \varepsilon_{1 i}, \varepsilon_{2 i}\right)$ is strictly concave in $(x, y)$ when $\varepsilon_{1 i}>0$ and $\varepsilon_{2 i}>0$ hold for $i \in\{a, b\}$. The inequalities in (39) can be expressed as $g\left(x_{a}, y_{a} \mid a, 0,0\right)<$ $g\left(x_{a}, y_{a} \mid b, 0,0\right)$ and $g\left(x_{b}, y_{b} \mid b, 0,0\right)<g\left(x_{b}, y_{b} \mid a, 0,0\right)$. Due to continuity of $g$, these strict inequalities remain true if $\varepsilon_{1 i}$ and $\varepsilon_{2 i}$ are sufficiently small positive numbers instead of 0 . It is therefore possible to find positive numbers $\bar{\varepsilon}_{1 i}$ and $\bar{\varepsilon}_{2 i}$ such that $g\left(x, y \mid i, \bar{\varepsilon}_{1 i}, \bar{\varepsilon}_{2 i}\right)$ is strictly increasing and strictly concave with respect to $(x, y)$ for $i \in\{a, b\}$ and such that

$$
\begin{aligned}
& g\left(x_{a}, y_{a} \mid a, \bar{\varepsilon}_{1 a}, \bar{\varepsilon}_{2 a}\right)<g\left(x_{a}, y_{a} \mid b, \bar{\varepsilon}_{1 b}, \bar{\varepsilon}_{2 b}\right), \\
& g\left(x_{b}, y_{b} \mid b, \bar{\varepsilon}_{1 b}, \bar{\varepsilon}_{2 b}\right)<g\left(x_{b}, y_{b} \mid a, \bar{\varepsilon}_{1 a}, \bar{\varepsilon}_{2 a}\right)
\end{aligned}
$$

hold.

Step 3: We define the function $w: X \times Y \mapsto \mathbb{R}$ by

$$
w(x, y)=\min \left\{g\left(x, y \mid i, \bar{\varepsilon}_{1 i}, \bar{\varepsilon}_{2 i}\right) \mid i \in\{a, b\}\right\} .
$$

As a minimum of continuous, strictly increasing, and strictly concave functions, the function $w$ itself is also continuous, strictly increasing, and strictly concave. The inequalities stated in (40)-(41) imply furthermore that $w$ is continuously differentiable locally around the points $\left(x_{a}, y_{a}\right)$ and $\left(x_{b}, y_{b}\right)$ and that its partial derivatives at these points are given by $w_{1}\left(x_{i}, y_{i}\right)=$ $g_{1}\left(x_{i}, y_{i} \mid i, \bar{\varepsilon}_{1 i}, \bar{\varepsilon}_{2 i}\right)=w_{1 i}$ and $w_{2}\left(x_{i}, y_{i}\right)=g_{2}\left(x_{i}, y_{i} \mid i, \bar{\varepsilon}_{1 i}, \bar{\varepsilon}_{2 i}\right)=w_{2 i}$ for $i \in\{a, b\}$.

Proof of theorem 1 We define $c_{a}$ and $c_{b}$ by Eqs. (29)-(30). This yields strictly positive values if and only if conditions (5)-(6) hold. Condition (4) coincides with (31). It remains to show that there exists a function $u:[0, \bar{k}] \times[0,1] \mapsto \mathbb{R}$ satisfying Assumption 2 and conditions (32)-(34). Since the latter equations are homogeneous in the partial derivatives $u_{1}\left(c_{i}, 1-\ell_{i}\right)$ and $u_{2}\left(c_{i}, 1-\ell_{i}\right)$ for $i \in\{a, b\}$, we may normalize the function $u$ by setting $u_{1}\left(c_{a}, 1-\ell_{a}\right)=1$. Solving (32)-(34) under this normalization yields 


$$
\begin{aligned}
& u_{1}\left(c_{a}, 1-\ell_{a}\right)=1, \\
& u_{1}\left(c_{b}, 1-\ell_{b}\right)=\beta f_{1}\left(k_{a}, \ell_{a}\right), \\
& u_{2}\left(c_{a}, 1-\ell_{a}\right)=f_{2}\left(k_{a}, \ell_{a}\right), \\
& u_{2}\left(c_{b}, 1-\ell_{b}\right)=\beta f_{1}\left(k_{a}, \ell_{a}\right) f_{2}\left(k_{b}, \ell_{b}\right) .
\end{aligned}
$$

Now we apply Lemma 2 with $X=[0, \bar{k}], Y=[0,1], x_{i}=c_{i}$, and $y_{i}=1-\ell_{i}$ for $i \in\{a, b\}$. This shows that there exists a function $u:[0, \bar{k}] \times[0,1] \mapsto \mathbb{R}$ such that Assumption 2 is satisfied and such that the partial derivatives of $u$ at the points $\left(c_{a}, 1-\ell_{a}\right)$ and $\left(c_{b}, 1-\ell_{b}\right)$ exist and are given by (42)-(45) if and only if the inequality

$$
\left[1-\beta f_{1}\left(k_{a}, \ell_{a}\right)\right]\left(c_{b}-c_{a}\right)+\left[f_{2}\left(k_{a}, \ell_{a}\right)-\beta f_{1}\left(k_{a}, \ell_{a}\right) f_{2}\left(k_{b}, \ell_{b}\right)\right]\left(\ell_{a}-\ell_{b}\right)>0
$$

holds. We can use (29)-(30) to eliminate $c_{a}$ and $c_{b}$ from this condition, which yields

$$
\begin{aligned}
& f\left(k_{b}, \ell_{b}\right)+k_{b}-k_{a}+f_{2}\left(k_{a}, \ell_{a}\right) \ell_{a}-f\left(k_{a}, \ell_{a}\right)-f_{2}\left(k_{a}, \ell_{a}\right) \ell_{b} \\
& \quad+\beta f_{1}\left(k_{a}, \ell_{a}\right)\left[f\left(k_{a}, \ell_{a}\right)+k_{a}-k_{b}+f_{2}\left(k_{b}, \ell_{b}\right) \ell_{b}-f\left(k_{b}, \ell_{b}\right)\right. \\
& \left.\quad-f_{2}\left(k_{b}, \ell_{b}\right) \ell_{a}\right]>0 .
\end{aligned}
$$

Linear homogeneity of $f$ implies that $f_{2}\left(k_{i}, \ell_{i}\right) \ell_{i}-f\left(k_{i}, \ell_{i}\right)=-f_{1}\left(k_{i}, \ell_{i}\right) k_{i}$ for $i \in\{a, b\}$. Substituting this into the above formula, we obtain (7). This completes the proof of Theorem 1.

Proof of local asymptotic stability in Example 1 The dynamics of optimal allocations are determined by Eqs. (1) and (35)-(36). Linearizing these equations, we obtain

$$
\begin{aligned}
& \mathrm{d} c_{t}+\mathrm{d} k_{t+1}=f_{1}(t) \mathrm{d} k_{t}+f_{2}(t) \mathrm{d} \ell_{t}, \\
& u_{11}(t) \mathrm{d} c_{t}-u_{12}(t) \mathrm{d} \ell_{t}=\beta f_{1}(t+1)\left[u_{11}(t+1) \mathrm{d} c_{t+1}-u_{12}(t+1) \mathrm{d} \ell_{t+1}\right] \\
& \quad+\beta u_{1}(t+1)\left[f_{11}(t+1) \mathrm{d} k_{t+1}+f_{12}(t+1) \mathrm{d} \ell_{t+1}\right], \\
& f_{2}(t)\left[u_{11}(t) \mathrm{d} c_{t}-u_{12}(t) \mathrm{d} \ell_{t}\right]+u_{1}(t)\left[f_{12}(t) \mathrm{d} k_{t}+f_{22}(t) \mathrm{d} \ell_{t}\right]=u_{12}(t) \mathrm{d} c_{t}-u_{22}(t) \mathrm{d} \ell_{t},
\end{aligned}
$$

where we have shortened the notation by indicating evaluation of $f, u$, and their partial derivatives at period- $s$ arguments by $(s)$. We can use the third of these equations to express $\mathrm{d} \ell_{s}$ in terms of $\mathrm{d} k_{s}$ and $\mathrm{d} c_{s}$ for $s \in\{t, t+1\}$. Substituting these expressions into the first two equations and solving them for $\left(\mathrm{d} k_{t+1}, \mathrm{~d} c_{t+1}\right)$, we obtain

$$
\left(\begin{array}{l}
\mathrm{d} k_{t+1} \\
\mathrm{~d} c_{t+1}
\end{array}\right)=J\left(k_{t}, \ell_{t}, c_{t}\right)\left(\begin{array}{l}
\mathrm{d} k_{t} \\
\mathrm{~d} c_{t}
\end{array}\right),
$$

where $J\left(k_{t}, \ell_{t}, c_{t}\right) \in \mathbb{R}^{2 \times 2}$ is the Jacobian matrix at point $\left(k_{t}, \ell_{t}, c_{t}\right)$. Since capital is a predetermined variable while consumption is not, the periodic allocation defined in (3) is locally asymptotically stable if the matrix $\mathbf{J}:=J\left(k_{a}, \ell_{a}, c_{a}\right) J\left(k_{b}, \ell_{b}, c_{b}\right)$ has one stable eigenvalue and if the corresponding eigenvector $\left(z_{k}, z_{c}\right)^{\top}$ satisfies $z_{k} \neq 0 .{ }^{10}$

10 See Sorger (2015), theorem 3.7. 
We shall now verify that this is the case for the periodic optimal allocation constructed in Example 1 and Theorem 1.

From the construction of $u$ in the proofs of Theorem 1 and Lemma 2, we know that the first-order partial derivatives of the utility function are given by (42)-(45), whereas the second-order partial derivatives are $u_{11}\left(c_{i}, 1-\ell_{i}\right)=-2 \bar{\varepsilon}_{1 i}, u_{22}\left(c_{i}, 1-\right.$ $\left.\ell_{i}\right)=-2 \bar{\varepsilon}_{2 i}$, and $u_{12}\left(c_{i}, 1-\ell_{i}\right)=0$ for $i \in\{a, b\}$. Furthermore, from $f(k, \ell)=$ $(\alpha \beta)^{-1} k^{\alpha} \ell^{1-\alpha}, k_{a}=\gamma \ell_{a}$, and $k_{b}=\gamma^{-1} \ell_{b}$ we can easily compute all the first- and second-order partial derivatives of the production function at the two points $\left(k_{a}, \ell_{a}\right)$ and $\left(k_{b}, \ell_{b}\right)$. Using all of these results and choosing $\bar{\varepsilon}_{2 a}=\bar{\varepsilon}_{2 b}=\varepsilon^{3}, \bar{\varepsilon}_{1 b}=\varepsilon^{2}$, and $\bar{\varepsilon}_{1 a}=\varepsilon$, we obtain

$$
\lim _{\varepsilon \rightarrow 0} \mathbf{J}=\left(\begin{array}{cc}
1 /(\alpha \beta)^{2}-\gamma^{\alpha-1} /(\alpha \beta) \\
0 & \alpha^{2}
\end{array}\right)
$$

This matrix has the stable eigenvalue $\alpha^{2}$ with corresponding eigenvector

$$
\left(\begin{array}{c}
\alpha \beta \gamma^{\alpha-1} \\
1-\alpha^{4} \beta^{2}
\end{array}\right)
$$

Since the eigenvalues and eigenvectors of a matrix depend continuously on the entries of the matrix, it follows that $\mathbf{J}$ has a stable eigenvalue and that the corresponding eigenvector has a nonzero first component also if $\varepsilon$ is positive but sufficiently small. For such a choice of $\varepsilon$, it follows therefore that the periodic allocation constructed in Example 1 is locally asymptotically stable.

Proof of theorem 2 (a) Suppose to the contrary that the sequence defined by (3) is an optimal allocation and that the utility function $u$ is additively separable and locally differentiable along this allocation. Then it follows that conditions (29)-(31) and (42)(45) hold (after scaling the utility function such that $u_{1}\left(c_{a}, 1-\ell_{a}\right)=1$ ). Since $u(c, 1-\ell)=v(c)+q(1-\ell)$, the latter four conditions can be written as

$$
\begin{aligned}
& v^{\prime}\left(c_{a}\right)=1, \\
& v^{\prime}\left(c_{b}\right)=\beta f_{1}\left(k_{a}, \ell_{a}\right), \\
& q^{\prime}\left(1-\ell_{a}\right)=f_{2}\left(k_{a}, \ell_{a}\right), \\
& q^{\prime}\left(1-\ell_{b}\right)=\beta f_{1}\left(k_{a}, \ell_{a}\right) f_{2}\left(k_{b}, \ell_{b}\right) .
\end{aligned}
$$

Without loss of generality, we can assume $c_{a} \leq c_{b}$. Concavity of $v$ and (46)-(47) imply that $\beta f_{1}\left(k_{a}, \ell_{a}\right) \leq 1$. Because of (31) we obtain $\beta f_{1}\left(k_{b}, \ell_{b}\right) \geq 1$ and, hence, $f_{1}\left(k_{a}, \ell_{a}\right) \leq f_{1}\left(k_{b}, \ell_{b}\right)$. Concavity and linear homogeneity of $f$ imply that $k_{a} / \ell_{a} \geq$ $k_{b} / \ell_{b}$ and $f_{2}\left(k_{a}, \ell_{a}\right) \geq f_{2}\left(k_{b}, \ell_{b}\right)$. Combining these results, we get

$$
f_{2}\left(k_{a}, \ell_{a}\right) \geq \beta f_{1}\left(k_{a}, \ell_{a}\right) f_{2}\left(k_{b}, \ell_{b}\right) .
$$


Together with (48)-(49) and the concavity of $q$, this shows that $\ell_{b} \leq \ell_{a}$. Since it is assumed that $k_{a} \neq k_{b}$ and since we already know that $k_{a} / \ell_{a} \geq k_{b} / \ell_{b}$, it follows that $k_{a}>k_{b}$. Putting everything into (29)-(30), it follows that $k_{b}=f\left(k_{a}, \ell_{a}\right)-c_{a} \geq$ $f\left(k_{b}, \ell_{b}\right)-c_{b}=k_{a}$. This is a contradiction to $k_{a}>k_{b}$ and the proof is complete.

(b) The proof is similar to that of De Hek (1998), proposition 2.4, which is why we omit some technical details. The Bellman equation for the problem under consideration is

$$
V(k)=\max _{\ell, k^{\prime}}\left\{u\left(f(k, \ell)-k^{\prime}, 1-\ell\right)+\beta V\left(k^{\prime}\right) \mid \ell \in[0,1], k^{\prime} \in[0, f(k, \ell)]\right\},
$$

where $V:[0, \bar{k}] \mapsto \mathbb{R}$ is the optimal value function. This function is differentiable, strictly increasing, and strictly concave. Due to strict concavity and boundedness assumptions, there exists a unique solution to the optimization problem on the righthand side of this equation, which we denote by $\left(\ell, k^{\prime}\right)=(g(k), h(k))$. The first-order conditions are

$$
\begin{aligned}
& u_{1}(f(k, g(k))-h(k), 1-g(k)) f_{2}(k, g(k))-u_{2}(f(k, g(k))-h(k), 1-g(k))=0, \\
& -u_{1}(f(k, g(k))-h(k), 1-g(k))+\beta V^{\prime}(h(k))=0 .
\end{aligned}
$$

Totally differentiating these equations with respect to $k$, we obtain

$$
\begin{aligned}
& \left(u_{11} f_{2}-u_{12}\right)\left[f_{1}+f_{2} g^{\prime}(k)-h^{\prime}(k)\right]+u_{1}\left[f_{12}+f_{22} g^{\prime}(k)\right] \\
& -u_{12} f_{2} g^{\prime}(k)+u_{22} g^{\prime}(k)=0,-u_{11}\left[f_{1}+f_{2} g^{\prime}-h^{\prime}(k)\right]+u_{12} g^{\prime}(k)+\beta V^{\prime \prime} h^{\prime}(k)=0,
\end{aligned}
$$

where the functional arguments of $f, u$, and $V$ as well as their derivatives have been omitted for ease of notation. Solving these two equations for $h^{\prime}(k)$ and $g^{\prime}(k)$, one obtains

$$
h^{\prime}(k)=\frac{u_{1} f_{12}\left(u_{12}-f_{2} u_{11}\right)+f_{1}\left(u_{11} u_{22}-u_{12}^{2}+u_{1} u_{11} f_{22}\right)}{u_{11} u_{22}-u_{12}^{2}+\beta f_{2}^{2} u_{11} V^{\prime \prime}-2 \beta f_{2} u_{12} V^{\prime \prime}+\beta u_{22} V^{\prime \prime}+u_{1} f_{22}\left(u_{11}+\beta V^{\prime \prime}\right)} .
$$

It is easy to see that under Assumptions 1-3 and those stated in the theorem both the numerator and the denominator of this expression are strictly positive. Hence, the policy function $h$ must be non-decreasing. Because optimal allocations satisfy $k_{t+1}=$ $h\left(k_{t}\right)$, it follows therefore that they must be monotonic. ${ }^{11}$ This completes the proof.

Lemma 3 Let $(f, u, \beta)$ be an economy satisfying Assumptions 1-3 and suppose that there exist real numbers $k_{a}, k_{b}, k_{c}, \ell_{a}, \ell_{b}, \ell_{c}, c_{a}, c_{b}$, and $c_{c}$ such that $k_{a} \neq k_{b}, k_{b} \neq k_{c}$, $k_{a} \neq k_{c}$, and $\left(k_{i}, \ell_{i}, c_{i}\right) \in(0, \bar{k}) \times(0,1) \times(0, \bar{k})$ for $i \in\{a, b, c\}$ hold and such that the utility function $u$ is continuously differentiable locally around each of the three points $\left(c_{a}, 1-\ell_{a}\right),\left(c_{b}, 1-\ell_{b}\right)$, and $\left(c_{c}, 1-\ell_{c}\right)$.

11 See Sorger (2015), lemma 4.1. 
(a) The sequence $\left(k_{t}, \ell_{t}, c_{t}\right)_{t=0}^{+\infty}$ defined by (18) is an optimal allocation for the economy $(f, u, \beta)$ if and only if the following conditions hold:

$$
\begin{aligned}
& c_{a}+k_{b}=f\left(k_{a}, \ell_{a}\right), c_{b}+k_{c}=f\left(k_{b}, \ell_{b}\right) c_{c}+k_{a}=f\left(k_{c}, \ell_{c}\right), \\
& \beta^{3} f_{1}\left(k_{a}, \ell_{a}\right) f_{1}\left(k_{b}, \ell_{b}\right) f_{1}\left(k_{c}, \ell_{c}\right)=1, \\
& u_{1}\left(c_{b}, 1-\ell_{b}\right)=\beta f_{1}\left(k_{c}, \ell_{c}\right) u_{1}\left(c_{c}, 1-\ell_{c}\right), \\
& u_{1}\left(c_{c}, 1-\ell_{c}\right)=\beta f_{1}\left(k_{a}, \ell_{a}\right) u_{1}\left(c_{a}, 1-\ell_{a}\right), \\
& u_{1}\left(c_{i}, 1-\ell_{i}\right) f_{2}\left(k_{i}, \ell_{i}\right)=u_{2}\left(c_{i}, 1-\ell_{i}\right) \text { for } i \in\{a, b, c\} .
\end{aligned}
$$

(b) If the sequence $\left(k_{t}, \ell_{t}, c_{t}\right)_{t=0}^{+\infty}$ defined by (18) is an optimal allocation for the economy $(f, u, \beta)$, then it follows that the three points $\left(c_{a}, 1-\ell_{a}\right),\left(c_{b}, 1-\ell_{b}\right)$ and $\left(c_{c}, 1-\ell_{c}\right)$ are mutually different from each other.

Proof (a) The proof of this part is completely analogous to that of Lemma 1(a) and is therefore omitted.

(b) Since the economy satisfies Assumptions 1-3, standard arguments from dynamic programming imply that optimal allocations exist and that the optimal value function $V:[0, \bar{k}] \mapsto \mathbb{R}$ is bounded, continuous, and strictly concave. Moreover, it holds for $i \in\{a, b, c\}$ that

$$
\left(c_{i}, \ell_{i}\right)=\operatorname{argmax}\left\{u(c, 1-\ell)+\beta V\left(f\left(k_{i}, \ell\right)-c\right) \mid c \in\left[0, f\left(k_{i}, \ell\right)\right], \ell \in[0,1]\right\} .
$$

Since $\left(k_{i}, \ell_{i}, c_{i}\right) \in(0, \bar{k}) \times(0,1) \times(0, \bar{k})$ holds for $i \in\{a, b, c\}$, it follows that

$$
0 \in\left\{u_{1}\left(c_{i}, 1-\ell_{i}\right)-\beta p \mid p \in \partial V\left(f\left(k_{i}, \ell_{i}\right)-c_{i}\right)\right\},
$$

where $\partial V(k)$ denotes the subdifferential of the strictly concave function $V$ at $k .^{12}$ Now suppose that $\left(c_{a}, 1-\ell_{a}\right)=\left(c_{b}, 1-\ell_{b}\right)=(\bar{c}, 1-\bar{\ell})$. The above condition together with (50) implies that

$$
u_{1}(\bar{c}, 1-\bar{\ell}) / \beta \in \partial V\left(k_{b}\right) \cap \partial V\left(k_{c}\right)
$$

Because $V$ is strictly concave and $k_{b} \neq k_{c}$ by assumption, the right-hand side of this formula is the empty set. Hence, we obtain a contradiction to the assumption $\left(c_{a}, 1-\ell_{a}\right)=\left(c_{b}, 1-\ell_{b}\right)$, which proves this part of the lemma.

Lemma 4 Let $X$ and $Y$ be non-empty and compact intervals on the real line and let $x_{a}, x_{b}, x_{c}, y_{a}, y_{b}$, and $y_{c}$ be real numbers such that $x_{i} \in$ int $X$ and $y_{i} \in$ int $Y$ hold for $i \in\{a, b, c\}$ and such that the three points $\left(x_{a}, y_{a}\right),\left(x_{b}, y_{b}\right)$, and $\left(x_{c}, y_{c}\right)$ are mutually different from each other. Furthermore, let $w_{1 a}, w_{1 b}, w_{1 c}, w_{2 a}, w_{2 b}$, and $w_{2 c}$ be positive real numbers. The following two statements are equivalent:

\footnotetext{
12 In parts of the literature, the terminology "subdifferential" is only used for convex functions, whereas it is replaced by "superdifferential" in the case of concave functions.
} 
(a) There exists a function $w: X \times Y \mapsto \mathbb{R}$ which is continuous, strictly increasing, and strictly concave and which is continuously differentiable locally at the points $\left(x_{a}, y_{a}\right)$, $\left(x_{b}, y_{b}\right)$, and $\left(x_{c}, y_{c}\right)$ with partial derivatives $w_{1}\left(x_{i}, y_{i}\right)=w_{1 i}$ and $w_{2}\left(x_{i}, y_{i}\right)=w_{2 i}$ for $i \in\{a, b, c\}$.

(b) It holds that

$$
\begin{gathered}
\left(w_{1 b}-w_{1 a}\right)\left(x_{a}-x_{b}\right)+\left(w_{2 b}-w_{2 a}\right)\left(y_{a}-y_{b}\right)>0, \\
\left(w_{1 c}-w_{1 b}\right)\left(x_{b}-x_{c}\right)+\left(w_{2 c}-w_{2 b}\right)\left(y_{b}-y_{c}\right)>0, \\
\left(w_{1 c}-w_{1 a}\right)\left(x_{a}-x_{c}\right)+\left(w_{2 c}-w_{2 a}\right)\left(y_{a}-y_{c}\right)>0, \\
w_{1 a}\left(x_{b}-x_{a}\right)+w_{1 b}\left(x_{c}-x_{b}\right)+w_{1 c}\left(x_{a}-x_{c}\right) \\
\quad>w_{2 a}\left(y_{a}-y_{b}\right)+w_{2 b}\left(y_{b}-y_{c}\right)+w_{2 c}\left(y_{c}-y_{a}\right), \\
w_{1 a}\left(x_{c}-x_{a}\right)+w_{1 b}\left(x_{a}-x_{b}\right)+w_{1 c}\left(x_{b}-x_{c}\right) \\
\quad>w_{2 a}\left(y_{a}-y_{c}\right)+w_{2 b}\left(y_{b}-y_{a}\right)+w_{2 c}\left(y_{c}-y_{b}\right) .
\end{gathered}
$$

Proof We first prove that (a) implies (b). Since the three points $\left(x_{a}, y_{a}\right),\left(x_{b}, y_{b}\right)$, and $\left(x_{c}, y_{c}\right)$ are mutually different, it follows from strict concavity of $w$ that

$$
\begin{aligned}
& w\left(x_{a}, y_{a}\right)<w\left(x_{b}, y_{b}\right)+w_{1 b}\left(x_{a}-x_{b}\right)+w_{2 b}\left(y_{a}-y_{b}\right), \\
& w\left(x_{a}, y_{a}\right)<w\left(x_{c}, y_{c}\right)+w_{1 c}\left(x_{a}-x_{c}\right)+w_{2 c}\left(y_{a}-y_{c}\right), \\
& w\left(x_{b}, y_{b}\right)<w\left(x_{a}, y_{a}\right)+w_{1 a}\left(x_{b}-x_{a}\right)+w_{2 a}\left(y_{b}-y_{a}\right), \\
& w\left(x_{b}, y_{b}\right)<w\left(x_{c}, y_{c}\right)+w_{1 c}\left(x_{b}-x_{c}\right)+w_{2 c}\left(y_{b}-y_{c}\right), \\
& w\left(x_{c}, y_{c}\right)<w\left(x_{a}, y_{a}\right)+w_{1 a}\left(x_{c}-x_{a}\right)+w_{2 a}\left(y_{c}-y_{a}\right), \\
& w\left(x_{c}, y_{c}\right)<w\left(x_{b}, y_{b}\right)+w_{1 b}\left(x_{c}-x_{b}\right)+w_{2 b}\left(y_{c}-y_{b}\right) .
\end{aligned}
$$

Combining (60) and (62), one obtains (55). In the same way, one gets (56) from (63) and (65) and one gets (57) from (61) and (64). Adding (61), (62), and (65) yields (59), and by adding (60), (63), and (64) one obtains (58).

The proof that (b) implies (a) is split up in the same three steps as the proof of the corresponding part in Lemma 2.

Step 1: We first prove that there exist real numbers $w_{a}, w_{b}$, and $w_{c}$ such that the inequalities

$$
\begin{aligned}
& w_{1 b}\left(x_{b}-x_{a}\right)+w_{2 b}\left(y_{b}-y_{a}\right)<w_{b}-w_{a}<w_{1 a}\left(x_{b}-x_{a}\right)+w_{2 a}\left(y_{b}-y_{a}\right), \\
& w_{1 c}\left(x_{c}-x_{b}\right)+w_{2 c}\left(y_{c}-y_{b}\right)<w_{c}-w_{b}<w_{1 b}\left(x_{c}-x_{b}\right)+w_{2 b}\left(y_{c}-y_{b}\right), \\
& w_{1 c}\left(x_{c}-x_{a}\right)+w_{2 c}\left(y_{c}-y_{a}\right)<w_{c}-w_{a}<w_{1 a}\left(x_{c}-x_{a}\right)+w_{2 a}\left(y_{c}-y_{a}\right)
\end{aligned}
$$


hold. To this end, we define the real numbers

$$
\begin{aligned}
& A_{b a}=w_{1 b}\left(x_{b}-x_{a}\right)+w_{2 b}\left(y_{b}-y_{a}\right), \quad B_{b a}=w_{1 a}\left(x_{b}-x_{a}\right)+w_{2 a}\left(y_{b}-y_{a}\right), \\
& A_{c b}=w_{1 c}\left(x_{c}-x_{b}\right)+w_{2 c}\left(y_{c}-y_{b}\right), B_{c b}=w_{1 b}\left(x_{c}-x_{b}\right)+w_{2 b}\left(y_{c}-y_{b}\right), \\
& A_{c a}=w_{1 c}\left(x_{c}-x_{a}\right)+w_{2 c}\left(y_{c}-y_{a}\right), \quad B_{c a}=w_{1 a}\left(x_{c}-x_{a}\right)+w_{2 a}\left(y_{c}-y_{a}\right),
\end{aligned}
$$

and the open intervals $I_{b a}=\left(A_{b a}, B_{b a}\right), I_{c b}=\left(A_{c b}, B_{c b}\right)$, and $I_{c a}=$ $\left(A_{c a}, B_{c a}\right)$. It follows from (55)-(57) that all three of these intervals are non-empty. Furthermore, it follows from (58)-(59) that $A_{b a}+A_{c b}<$ $B_{c a}$ and $A_{c a}<B_{b a}+B_{c b}$. This, in turn, implies that

$$
\left\{\delta_{b a}+\delta_{c b} \mid \delta_{b a} \in I_{b a}, \delta_{c b} \in I_{c b}\right\} \cap I_{c a} \neq \emptyset .
$$

Consequently, there exist real numbers $\delta_{b a} \in I_{b a}, \delta_{c b} \in I_{c b}$, and $\delta_{c a} \in I_{c a}$ such that $\delta_{c a}=\delta_{b a}+\delta_{c b}$. Let $w_{a}$ be an arbitrary real number and define $w_{b}=w_{a}+\delta_{b a}$ and $w_{c}=w_{a}+\delta_{c a}$. Then it follows that $w_{b}-w_{a}=\delta_{b a} \in I_{b a}, w_{c}-w_{b}=\delta_{c a}-\delta_{b a}=\delta_{c b} \in I_{c b}$, and $w_{c}-w_{a}=\delta_{c a} \in I_{c a}$. Obviously, this is equivalent to (66)-(68).

Steps 2 and 3: Since these steps are completely analogous to the corresponding steps in the proof of Lemma 2, we omit many details. One starts by defining for $i \in\{a, b, c\}$ and all $\varepsilon \in \mathbb{R}_{+}$the quadratic polynomial $g(\cdot, \cdot \mid i, \varepsilon)$ : $X \times Y \mapsto \mathbb{R}$ by

$$
g(x, y \mid i, \varepsilon)=w_{i}+w_{1 i}\left(x-x_{i}\right)+w_{2 i}\left(y-y_{i}\right)-\varepsilon\left[\left(x-x_{i}\right)^{2}+\left(y-y_{i}\right)^{2}\right] .
$$

If $\varepsilon$ is positive but sufficiently small, $g(\cdot, \cdot \mid i, \varepsilon)$ is strictly increasing and strictly concave for $i \in\{a, b, c\}$. Then, one defines the function $w: X \times Y \mapsto \mathbb{R}$ by

$$
w(x, y)=\min \{g(x, y \mid i, \varepsilon) \mid i \in\{a, b, c\}\} .
$$

If $\varepsilon$ is positive but sufficiently small, then it follows that $w$ is a continuous, strictly increasing, and strictly concave function. Furthermore, the inequalities in (66)-(68) ensure that $w$ is continuously differentiable locally around the three points $\left(x_{a}, y_{a}\right),\left(x_{b}, y_{b}\right)$, and $\left(x_{c}, y_{c}\right)$ and that its partial derivatives at these points are given by

$$
w_{1}\left(x_{i}, y_{i}\right)=g_{1}\left(x_{i}, y_{i} \mid i, \varepsilon\right)=w_{1 i}
$$

and

$$
w_{2}\left(x_{i}, y_{i}\right)=g_{2}\left(x_{i}, y_{i} \mid i, \varepsilon\right)=w_{2 i}
$$

for $i \in\{a, b, c\}$. This completes the proof of the lemma. 
Proof of theorem 3 We define $c_{a}, c_{b}$, and $c_{c}$ by the equations in (50). This yields strictly positive values if and only if conditions (20)-(22) hold. Condition (19) coincides with (51). It remains to show that there exists a function $u:[0, \bar{k}] \times[0,1] \mapsto \mathbb{R}$ satisfying Assumption 2 and conditions (52)-(54). Since the latter equations are homogeneous in the partial derivatives $u_{1}\left(c_{i}, 1-\ell_{i}\right)$ and $u_{2}\left(c_{i}, 1-\ell_{i}\right)$ for $i \in\{a, b, c\}$, we may normalize the function $u$ by setting $u_{1}\left(c_{a}, 1-\ell_{a}\right)=1$. Solving (52)-(54) under this normalization yields

$$
\begin{aligned}
& u_{1}\left(c_{a}, 1-\ell_{a}\right)=u_{1 a}:=1, \\
& u_{1}\left(c_{b}, 1-\ell_{b}\right)=u_{1 b}:=\beta^{2} f_{1}\left(k_{a}, \ell_{a}\right) f_{1}\left(k_{c}, \ell_{c}\right), \\
& u_{1}\left(c_{c}, 1-\ell_{c}\right)=u_{1 c}:=\beta f_{1}\left(k_{a}, \ell_{a}\right), \\
& u_{2}\left(c_{a}, 1-\ell_{a}\right)=u_{2 a}:=f_{2}\left(k_{a}, \ell_{a}\right), \\
& u_{2}\left(c_{b}, 1-\ell_{b}\right)=u_{2 b}:=\beta^{2} f_{1}\left(k_{a}, \ell_{a}\right) f_{1}\left(k_{c}, \ell_{c}\right) f_{2}\left(k_{b}, \ell_{b}\right), \\
& u_{2}\left(c_{c}, 1-\ell_{c}\right)=u_{2 c}:=\beta f_{1}\left(k_{a}, \ell_{a}\right) f_{2}\left(k_{c}, \ell_{c}\right) .
\end{aligned}
$$

Now we apply Lemma 4 with $X=[0, \bar{k}], Y=[0,1], x_{i}=c_{i}$, and $y_{i}=1-\ell_{i}$ for $i \in\{a, b, c\}$. This shows that there exists a function $u:[0, \bar{k}] \times[0,1] \mapsto \mathbb{R}$ such that Assumption 2 is satisfied and such that the partial derivatives of $u$ at the points $\left(c_{a}, 1-\ell_{a}\right),\left(c_{b}, 1-\ell_{b}\right)$, and $\left(c_{c}, 1-\ell_{c}\right)$ exist and are given by (69)-(74) if and only if the inequalities

$$
\begin{aligned}
& \left(u_{1 b}-u_{1 a}\right)\left(c_{a}-c_{b}\right)+\left(u_{2 b}-u_{2 a}\right)\left(\ell_{b}-\ell_{a}\right)>0, \\
& \left(u_{1 c}-u_{1 b}\right)\left(c_{b}-c_{c}\right)+\left(u_{2 c}-u_{2 b}\right)\left(\ell_{c}-\ell_{b}\right)>0, \\
& \left(u_{1 c}-u_{1 a}\right)\left(c_{a}-c_{c}\right)+\left(u_{2 c}-u_{2 a}\right)\left(\ell_{c}-\ell_{a}\right)>0, \\
& u_{1 a}\left(c_{b}-c_{a}\right)+u_{1 b}\left(c_{c}-c_{b}\right)+u_{1 c}\left(c_{a}-c_{c}\right) \\
& \quad>u_{2 a}\left(\ell_{b}-\ell_{a}\right)+u_{2 b}\left(\ell_{c}-\ell_{b}\right)+u_{2 c}\left(\ell_{a}-\ell_{c}\right), \\
& u_{1 a}\left(c_{c}-c_{a}\right)+u_{1 b}\left(c_{a}-c_{b}\right)+u_{1 c}\left(c_{b}-c_{c}\right) \\
& \quad>u_{2 a}\left(\ell_{c}-\ell_{a}\right)+u_{2 b}\left(\ell_{a}-\ell_{b}\right)+u_{2 c}\left(\ell_{b}-\ell_{c}\right)
\end{aligned}
$$

hold, where the numbers $u_{1 i}$ and $u_{2 i}$ for $i \in\{a, b, c\}$ are defined in (69)-(74). We can use (50) to eliminate $c_{a}, c_{b}$, and $c_{c}$ from these conditions, and we can use linear homogeneity of the production function to replace $f_{2}\left(k_{i}, \ell_{i}\right) \ell_{i}-f\left(k_{i}, \ell_{i}\right)$ by $-f_{1}\left(k_{i}, \ell_{i}\right) k_{i}$ for $i \in\{a, b, c\}$. This leads to conditions (23)-(27) and the proof of Theorem 3 is complete.

\section{References}

Benhabib, J., Farmer, R.E.A.: Indeterminacy and increasing returns. J. Econ. Theory 63, 19-41 (1994) Collet, P., Eckmann, J.-P.: Iterated Maps on the Interval as Dynamical Systems. Birkhäuser, Basel (1980) De Hek, P.: An aggregative model of capital accumulation with leisure-dependent utility. J. Econ. Dyn. Control 23, 255-276 (1998)

De Melo, W., Van Strien, S.: One-Dimensional Dynamics. Springer, Berlin (1993)

Hartl, R.F.: A simple proof of the monotonicity of the state trajectories in autonomous control problems. J. Econ. Theory 41, 211-215 (1987) 
Kamihigashi, T.: Multiple interior steady states in the Ramsey model with elastic labor supply. Int. J. Econ. Theory 11, 25-37 (2015)

Li, T.Y., Yorke, J.A.: Period three implies chaos. Am. Math. Mon. 82, 985-992 (1975)

Miao, J.: Economic Dynamics in Discrete Time. MIT Press, Cambridge (2014)

Mitra, T.: An exact discount factor restriction for period-three cycles in dynamic optimization models. J. Econ. Theory 69, 281-305 (1996)

Nishimura, K., Yano, M.: On the least upper bound of discount factors that are compatible with optimal period-three cycles. J. Econ. Theory 69, 306-333 (1996)

Sarkovskii, A.N.: Coexistence of cycles of a continuous map of a line into itself. Ukrains'kyi Matematychnyi Zhurnal 16, 61-71 (1964)

Sorger, G.: Period three implies heavy discounting. Math. Oper. Res. 19, 1007-1022 (1994)

Sorger, G.: Income and wealth distribution in a simple model of growth. Econ. Theor. 16, $23-42$ (2000)

Sorger, G.: Dynamic Economic Analysis: Deterministic Models in Discrete Time. Cambridge University Press, Cambridge (2015)

Stokey, N., Lucas Jr., R.E.: Recursive Methods in Economic Dynamics. Harvard University Press, Cambridge (1989) 